\title{
THE FIRST ORDER THEORY OF $N$-COLORABLE GRAPHS ${ }^{1}$ BY
}

\author{
WILLIAM H. WHEELER
}

\begin{abstract}
Every $\boldsymbol{N}$-colorable graph without loops or multiple edges is a substructure of a direct power of a particular, finite, $\mathrm{N}$-coloarable graph. Consequently, the class of $N$-colorable graphs without loops or endpoints can be recursively axiomatized by a first order, universal Horn theory. This theory has a model-companion which has a primitive recursive elimination of quantifiers and is decidable, complete, $\boldsymbol{N}_{0}$-categorical, and independent. The $N$-colorable graphs without loops or multiple edges which have a proper, prime model extension for the model-companion are precisely the finite, amalgamation bases.
\end{abstract}

A graph consists of a set of vertices and a set of edges each of which joins two vertices. A graph is $N$-colorable, or more precisely vertex $N$-colorable, if there is an assignment of the numbers $1,2, \ldots, N$ to the vertices of the graph such that distinct numbers are assigned to adjacent vertices. The first order theory of $N$-colorable graphs is the subject of this paper.

The theory of $N$-colorable graphs is introduced and investigated in $\$ 1$. There is a finite $N$-colorable graph with the property that each $N$-colorable graph is a substructure of a direct power of this particular graph. Consequently, the class of $N$-colorable graphs is axiomatized by a universal Horn theory. This theory is recursively axiomatizable, but it is not finitely axiomatizable if $N>1$.

The existence and properties of the model-companion for the theory of $N$-colorable graphs are established in $\$ 2$. The model-companion is complete and decidable and has a primitive recursive elimination of quantifiers. Furthermore, the model-companion is $\aleph_{0}$-categorical and independent. Finally, an $N$-colorable graph has a prime model extension for the modelcompanion if and only if it is finite and an amalgamation base.

This work extends the result in [9] that the class of 2-colorable graphs is axiomatized by a universal Horn theory which has a model-companion.

0. Preliminaries. This paper will follow the conventions set forth in [9]. First order formulas will be denoted by Greek letters $\varphi, \psi, \chi, \rho, \sigma, \theta, \Phi$ with or

Received by the editors February 22, 1977 and, in revised form, March 21, 1978.

AMS (MOS) subject classifications (1970). Primary 02H05, 02H13, 02H15; Secondary $05 \mathrm{C} 15$.

$\mathrm{Key}$ words and phrases. $\mathrm{N}$-colorable graph, first order theory, model-companion, elimination of quantifiers, $\aleph_{0}$-categoricity, prime model extension.

${ }^{1}$ This research was supported in part by NSF grant MCS76-07019. 
without subscripts or superscripts. The finite sequences $a_{1}, \ldots, a_{n}$, $v_{1}, \ldots, v_{n}, a_{1}^{i}, \ldots, a_{n}^{i}$ etc., will be denoted by $\mathbf{a}, \mathbf{v}, \mathbf{a}^{i}$, etc., respectively. The finite sequences $b_{1}, \ldots, b_{m}, x_{1}, \ldots, x_{m}, b_{1}^{i}, \ldots, b_{m}^{i}$, etc., will be denoted by $\mathbf{b}, \mathbf{x}, \mathbf{b}^{i}$, etc., respectively. The finite sequences $e_{1}, \ldots, e_{p}, y_{1}, \ldots, y_{p}$, etc., will be denoted by $\mathbf{e}, \mathbf{y}$, etc., respectively. The notation $\varphi\left(v_{1}, \ldots, v_{n}\right)$ or $\varphi(\mathbf{v})$ denotes that the variables occurring freely in $\varphi$ are among $v_{1}, \ldots, v_{n}$, and then $\varphi($ a) denotes the formula obtained by replacing each free occurrence of $v_{i}$ in $\varphi$ by an occurrence of $a_{i}$. A basic formula is one which is either atomic or the negation of an atomic formula.

The distinction between an element in a structure and a name for that element in the language of the structure will be blurred deliberately. Accordingly, a symbol $b$ will denote both a constant symbol in a language and an element of a structure with the convention that $b$ as a symbol in the language is the name of the element $b$ in the structure. The diagram of a structure $\mathfrak{M}$, denoted by $\operatorname{Diag}(\mathfrak{M})$, is the set of all basic sentences in the language of $\mathfrak{M}$ which are true in $\mathfrak{M}$. The positive diagram of $\mathfrak{M}$, denoted by $\operatorname{PDiag}(\boldsymbol{M})$, is the set of atomic sentences in the language of $\mathfrak{M}$ which are true in $\mathfrak{M}$.

A homomorphism from one structure into another is a mapping from the universe of the first into the universe of the second such that true atomic sentences are preserved, or in other words, the positive diagram of the first structure is preserved. A homomorphism is onto if it maps the universe of the first structure onto the universe of the second structure; the second structure is then the image of the first under the homomorphism. A homomorphism is an embedding if the true negated atomic sentences are preserved, or in other words, the diagram of the first structure is preserved.

The reader is referred to [8] for standard facts concerning first order logic, to [6] for the standard results on model-completeness, to [3] for facts concerning existentially complete structures and model-companions, and to [9] for the characterization of the universal theories with finite presentation decompositions which have model-companions and for the definitions relevant thereto.

A theory $T_{G R}$ axiomatizing the class of colorable graphs will be introduced in the next section. In this context, the phrases "graph", "subgraph", "homomorphism from one graph into another graph", etc., will be synonymous with "model of $T_{G R}$ ", "submodel relative to $T_{G R}$ ", etc.. An element of a graph will be called a vertex.

1. The theory of $N$-colorable graphs. Graph theorists, when discussing vertex colorings, restrict attention to graphs without multiple edges or loops. Accordingly, the appropriate first order language is a first order language with equality whose only nonlogical symbol is a binary predicate symbol $E$ (for edge). The class of graphs without multiple edges or loops is axiomatized 
by the axioms

$$
\begin{gathered}
\forall x \neg E(x, x), \\
\forall x \forall y[E(x, y) \rightarrow E(y, x)] .
\end{gathered}
$$

The theory with these two axioms will be denoted by $T_{G R}$. (See $\S 3$ for an alternative formalization.)

Two vertices $a$ and $b$ of a graph are said to be adjacent if $E(a, b)$ holds.

The complete $N$-graph for each $N>0$ is the graph $K_{N}$ with vertices $c_{1}$, $c_{2}, \ldots, c_{N}$ for which $E\left(c_{i}, c_{j}\right)$ holds whenever $i \neq j$. A graph $G$ is $N$-colorable if and only if there is a homomorphism from $G$ into $K_{N}$. Such a homomorphism will be called an $N$-coloring of $G$. Two $N$-colorings $f_{1}$ and $f_{2}$ of $G$ will be called equivalent if there is an automorphism $g$ of $K_{N}$ (i.e., $g$ is an embedding of $K_{N}$ onto itself) such that $f_{1}=g \circ f_{2}$. Clearly this is an equivalence relation on the set of $N$-colorings of $G$. Note that if $f_{2}$ is an $N$-coloring of $G$ and $a$ is a vertex of $G$, then there is an $N$-coloring $f_{1}$ equivalent to $f_{2}$ such that $f_{1}(a)=c_{1}$.

If $H$ is an $N$-colorable graph and there is a homomorphism from a graph $G$ into $H$, then $G$ is $N$-colorable also.

Define a graph $C C N$ (for cogenerator for $N$-colorable graphs) for each $N \geqslant 1$ as follows. The set of vertices of $C C N$ is $\left\{d_{i, j}: i=1, \ldots, n ; j=1,2\right\}$. Two distinct vertices $d_{i, j}$ and $d_{r, s}$ are adjacent in $C C N$, i.e. $E\left(d_{i, j}, d_{r, s}\right)$ holds, if and only if one of the following three conditions is satisfied:

(1) $j=s=1$ and $i \neq r$;

(2) $j=1, s=2$, and $i \neq r$; or

(3) $j=s=2, i \neq r, i \neq 1$, and $r \neq 1$.

$C C 1$ is the graph:

$$
\stackrel{\bullet}{d_{1,2}} \quad \stackrel{\bullet}{d_{1,1}}
$$

$C C 2$ is the graph:

$$
d_{1,2}^{\bullet} \cdot d_{2,1} \quad d_{1,1} \quad d_{1,2} .
$$

CC 3 is the graph:

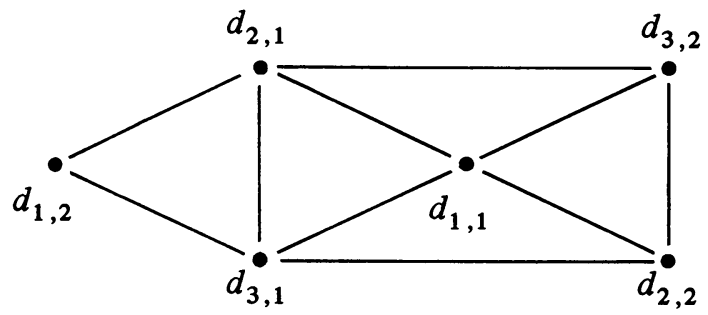


Proposition 1.1. The graph CCN is $N$-colorable.

Proof. Define a mapping $f$ from $C C N$ into $K_{N}$ by $f\left(d_{i, j}\right)=c_{i}$. If $d_{i, j}$ and $d_{r, s}$ are adjacent vertices of $C C N$, then $i \neq r$, so $f\left(d_{i, j}\right)=c_{i}$ and $f\left(d_{r, s}\right)=c_{r}$ are distinct vertices of $K_{N}$. Since distinct vertices of $K_{N}$ are adjacent, $f$ is a homomorphism.

The following theorem generalizes Proposition 8 of [9].

THEOREM 1.2. A graph is $N$-colorable if and only if it is a substructure of $a$ direct power of $C C N$.

Proof. If a graph $G$ is substructure of a direct power of $C C N$, then the projection map for each coordinate restricts to a homomorphism from $G$ into $C C N$. Since $C C N$ is $N$-colorable, $G$ is $N$-colorable.

Conversely suppose that $G$ is an $N$-colorable graph. Let $f$ be an $N$-coloring of $G$, that is, a homomorphism from $G$ into $K_{N}$. Define for each vertex $a$ of $G$ a homomorphism $f_{a}$ from $G$ into $C C N$ as follows. First, let $f^{\prime}$ be an $N$ coloring of $G$ which is equivalent to $f$ and for which $f^{\prime}(a)=c_{1}$. Define $f_{a}$ by $f_{a}(x)=d_{1,2}$ if $x=a, f_{a}(x)=d_{1,1}$ if $x \neq a$ and $f^{\prime}(x)=c_{1}, f_{a}(x)=d_{i, 1}$ if $x$ is adjacent to $a$ and $f^{\prime}(x)=c_{i}$, and $f_{a}(x)=d_{i, 2}$ if $x$ is not adjacent to $a$ and $f^{\prime}(x)=c_{i}$ for an $i \neq 1$. Note that the defining conditions for $f_{a}$ are mutually exclusive because $f^{\prime}$ cannot map a vertex which is adjacent to $a$ to $c_{1}$.

In order to verify that $f_{a}$ is a homomorphism, suppose that $c$ and $d$ are adjacent vertices of $G$.

Case 1. If $c=a$, then $f_{a}(c)=d_{1,2}$ and $f_{a}(d)=d_{i, 1}$, where $f^{\prime}(d)=c_{i}$ with $i \neq 1$. According to (2), $d_{1,2}$ and $d_{i, 1}$ for $i \neq 1$ are adjacent.

Case 2. Assume that $c \neq a$ and $d \neq a$.

Subcase (i). Suppose that both $c$ and $d$ are adjacent to $a$. Then $f^{\prime}(c)=c_{i}$ and $f^{\prime}(d)=c_{r}$ with $i \neq 1, r \neq 1$, and $i \neq r$, so $f_{a}(c)=d_{i, 1}$ and $f_{a}(d)=d_{r, 1}$ with $i \neq r$. According to (1), $d_{i, 1}$ and $d_{r, 1}$ for $i \neq r$ are adjacent.

Subcase (ii). Suppose that $c$ is adjacent to $a$ and $d$ is not adjacent to $a$. Then $f^{\prime}(c)=c_{i}$ with $i \neq 1$ and $f^{\prime}(d)=c_{r}$ with $r \neq i$. If $r=1$ then $f_{a}(c)=d_{i, 1}$ and $f_{a}(d)=d_{1,1}$ with $i \neq 1$, and $d_{i, 1}$ and $d_{1,1}$ for $i \neq 1$ are adjacent according to (1). If $r \neq 1$, then $f_{a}(c)=d_{i, 1}$ and $f_{a}(d)=d_{r, 2}$ with $i \neq r$, and $d_{i, 1}$ and $d_{r, 2}$ with $i \neq r$ are adjacent according to (2). In either alternative $f_{a}(c)$ and $f_{a}(d)$ are adjacent.

Subcase (iii). Suppose that neither $c$ nor $d$ is adjacent to $a$ and that $f^{\prime}(c) \neq c_{1}$. Then $f^{\prime}(c)=c_{i}$ and $f^{\prime}(d)=c_{r}$ with $i \neq 1$ and $r \neq i$. If $r=1$, then $f_{a}(c)=d_{i, 2}$ and $f_{a}(d)=d_{1,1}$ with $i \neq 1$, and $d_{i, 2}$ and $d_{1,1}$ for $i \neq 1$ are adjacent according to (2). If $r \neq 1$, then $f_{a}(c)=d_{i, 2}$ and $f_{a}(d)=d_{r, 2}$ with $i \neq r, i \neq 1$, and $r \neq 1$, in which case $d_{i, 2}$ and $d_{r, 2}$ are adjacent according to (3).

By symmetry these cases exhaust all possibilities. Thus $f_{a}(c)$ and $f_{a}(d)$ are adjacent, so $f_{a}$ is a homomorphism from $G$ into $C C N$. For notational 
convenience, let $C C N_{a}$ be a copy of $C C N$ and assume that $f_{a}$ maps $G$ into $\mathrm{CCN}_{a}$.

Let $F$ be the mapping $\Pi f_{a}$ from $G$ into $\Pi C C N_{a}$ where the index set for both products is the set of vertices of $G$. Then $F$ is a homomorphism since each $f_{a}$ is a homomorphism. That $F$ is an embedding must be verified. If $c$ and $d$ are distinct vertices of $G$, then $f_{c}(c) \neq d$, so $F(c) \neq F(d)$. If $c$ and $d$ are distinct, nonadjacent vertices of $G$, then $f_{c}(c)$ and $f_{c}(d)$ are distinct, nonadjacent vertices of $C C N_{c}$, so $F(c)$ and $F(d)$ are distinct, nonadjacent vertices. Thus, $F$ embeds $G$ as a substructure of a direct power of $C C N$.

Let $T_{C C N}$ be the universal theory of $C C N$ (in the language of $T_{G R}$ ). Let $\left(T_{C C N}\right)_{U H}$ be the collection of universal Horn consequences of $T_{C C N}$.

COROLlaRY 1.3. The class of $N$-colorable graphs is precisely the class of models of the theory $\left(T_{C C N}\right)_{U H}$.

Proof. This follows from Theorem 1.2 and Proposition 7 of [9].

THEOREM 1.4. The theory $\left(T_{C C N}\right)_{U H}$ of $N$-colorable graphs is recursively axiomatized.

Proof. The theory $T_{C C N}$ is decidable, because it has only finitely many models (up to isomorphism) each of which is finite, namely, the substructures of $C C N$. Consequently, the set $S$ of formulas which are valid in all direct products of models of $T_{C C N}$ is a recursive set (Theorem 6.9 of [2]). The collection $\left(T_{C C N}\right)_{U H}$ is just the set of universal Horn formulas which are in $S$, so $\left(T_{C C N}\right)_{U H}$ is a recursive set of formulas.

THEOREM 1.5. The theory $\left(T_{C C N}\right)_{U H}$ for $N \geqslant 2$ is not finitely axiomatizable.

Proof. If the theory $\left(T_{C C N}\right)_{U H}$ were finitely axiomatizable, then the class of $(N+1)$-colorable but not $N$-colorable graphs would be axiomatizable. But this would contradict Theorem 6.3 of [10].

That the class of $N$-colorable graphs is axiomatized by a recursive set of universal Horn formulas (Corollary 1.3 and Theorem 1.4) can be proved in other ways. W. Taylor has noted that a theorem of Erdös and de Bruijn can be used to construct an appropriate set of axioms. Hajos' Theorem would suffice also for this purpose. In this paper Theorem 1.2 is necessary to establish the relationship between the results of this paper, in particular, Theorem 2.2, and a conjecture on the companionability of universal Horn theories in [9]. Using Theorem 1.2, once it is available, to prove Corollary 1.3 and Theorem 1.4 seems then more reasonable in this instance than beginning anew with the results of Erdös and de Bruijn or Hajos.

2. The model-companion of the theory of $N$-colorable graphs. The theory of $N$-colorable graphs has a model-companion. However, this model-companion 
is not a model-completion for $N \geqslant 2$, because the theory of $N$-colorable graphs for $N \geqslant 2$ does not have the amalgamation property. The modelcompanion is complete and decidable, has a primitive recursive elimination of quantifiers, and is $\aleph_{0}$-categorical and independent. An $N$-colorable graph has a prime model extension for the model-companion if and only if it is finite and an amalgamation base.

Henceforth, assume $N \geqslant 2$.

Two $N$-colorable graphs $G_{1}$ and $G_{2}$ can be amalgamated as $N$-colorable graphs over a common subgraph $H$ if there exist an $N$-colorable graph $G_{3}$ and embeddings $g_{1}$ of $G_{1}$ into $G_{3}$ and $g_{2}$ of $G_{2}$ into $G_{3}$ such that the following diagram commutes:

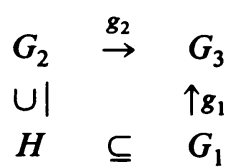

LEMMA 2.1. Two $N$-colorable graphs $G_{1}$ and $G_{2}$ can be amalgamated as $N$-colorable graphs over a common subgraph $H$ if and only if there are $N$-colorings of $G_{1}$ and $G_{2}$ which coincide on $H$.

Proof. The latter condition is clearly necessary.

Conversely, suppose there are homomorphisms $f_{1}$ from $G_{1}$ into $K_{N}$ and $f_{2}$ from $G_{2}$ into $K_{N}$ such that $f_{1}(a)=f_{2}(a)$ for all vertices $a$ of $H$. One may assume that $G_{1}$ and $G_{2}$ have only the vertices of $H$ in common. Let $G_{3}$ be the graph whose set of vertices is the union of the sets of vertices of $G_{1}$ and $G_{2}$ and in which two vertices are adjacent if and only if they are adjacent either in $G_{1}$ or in $G_{2}$. Define a mapping $f_{3}$ from $G_{3}$ into $K_{N}$ by $f_{3}(x)=f_{1}(x)$ if $x$ is in $G_{1}$ and $f_{3}(x)=f_{2}(x)$ if $x$ is in $G_{2}$. This mapping is well defined, since $f_{1}$ and $f_{2}$ coincide on $H$. The mapping $f$ is easily verified to be a homomorphism. Clearly, the following diagram commutes:

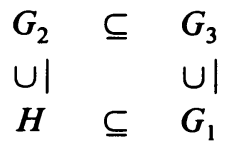

An $N$-colorable graph $H$ is called an amalgamation base if whenever $G_{1}$ and $G_{2}$ are $N$-colorable graphs containing $H$ as a subgraph, then $G_{1}$ and $G_{2}$ can be amalgamated as $N$-colorable graphs over $H$. The preceding lemma implies that $H$ is an amalgamation base if and only if all $N$-colorings of $H$ are equivalent.

Suppose that $G$ is an $N$-colorable graph and that $f$ is a homomorphism from $G$ into $K_{N}$. The $f$-fixation of $G$, denoted by $G_{f}$, is the graph whose set of vertices consists of the vertices of $G$ and new vertices $e_{1}, \ldots, e_{N}$ not in $G$ (where $p=N$ according to the convention in $\$ 0$ ) and in which two vertices $c$ 
and $d$ are adjacent if and only if they satisfy one of the following conditions: (1) $c$ and $d$ are in $G$ and are adjacent in $G$; (2) $c=e_{i}$ and $d=e_{j}$ with $i \neq j$; (3) $c$ is in $G, d=e_{i}$, and $f(c) \neq c_{i}$; or (4) $d$ is in $G, c=e_{i}$, and $f(d) \neq c_{i}$. The mapping $f^{\prime}$ from $G_{f}$ into $K_{N}$ defined by $f^{\prime}(x)=f(x)$ for $x$ in $G$ and $f\left(e_{i}\right)=c_{i}$ for $i=1, \ldots, N$ is a homomorphism from $G_{f}$ into $K_{N}$, so $G_{f}$ is $N$-colorable. Moreover, $G_{f}$ has only one $N$-coloring up to equivalence. Therefore $G_{f}$ is an amalgamation base. Suppose now that $a_{1}, \ldots, a_{n}$ are vertices of $G$. For each index $i, 1 \leqslant i \leqslant n$, let $V(i, f, G)=\left\{r: 1 \leqslant r \leqslant N\right.$ and $\left.f\left(a_{i}\right) \neq c_{r}\right\}$. Let $(\psi[G, f, \mathbf{a}])(\mathbf{v}, \mathbf{y})$ be the formula

$$
\left[\bigwedge_{r=1}^{N-1} \bigwedge_{s=r+1}^{N} E\left(y_{r}, y_{s}\right)\right] \wedge\left[\bigwedge_{i=1}^{n} \bigwedge_{r \in V(i, f, G)} E\left(v_{i}, y_{r}\right)\right] \text {. }
$$

Then $G_{f}$ satisfies $(\psi[G, f, \mathbf{a}])(\mathbf{a}, \mathbf{e})$.

Let $H$ be the graph consisting of two, nonadjacent vertices $a_{1}$ and $a_{2}$. This graph has two, nonequivalent $N$-colorings $f$ and $g$ defined by $f\left(a_{1}\right)=c_{1}$, $f\left(a_{2}\right)=c_{1}$ and $g\left(a_{1}\right)=c_{1}, g\left(a_{2}\right)=c_{2}$. Both graphs $H_{f}$ and $H_{g}$ contain $H$ as a subgraph. However, they do not have $N$-colorings which coincide on $H$, so they cannot be amalgamated over $H$. Thus, the theory of $N$-colorable graphs does not have the amalgamation property.

THEOREM 2.2. The theory of $N$-colorable graphs has a model-companion.

Proof. (This proof uses Theorem 1 of [9]. This theorem and the definitions of the technical terms used in this proof may be found in Appendix A. The proof itself is similar to the proof of Theorem 5 of [9]. The reader may wish to consult [9], particularly $\$ \S 2$ and 7 thereof, in order to gain insight into the methods of this proof.) The theory $\left(T_{C C N}\right)_{U H}$ of $N$-colorable graphs has finite presentations, and hence finite presentation decompositions, because it is universal Horn. This theory is coherent, because it is locally finite.

Consequently, according to Theorem 1 of [9], only the universal conservative homomorphism lifting property for finite presentations must be established. Only the following case need be considered, because $\left(T_{C C N}\right)_{U H}$ is locally finite. Let $G$ be an $N$-colorable graph with distinct vertices $a_{1}, \ldots, a_{n}$, $b_{1}, \ldots, b_{m}$ and no others. Let $H$ be the subgraph with vertices $a_{1}, \ldots, a_{n}$ and no others. Suppose that $\chi(\mathbf{v}, \mathbf{x})$ is a conjunction of negated atomic formulas and that $G$ satisfies $\chi(\mathbf{a}, \mathbf{b})$. The proof that $\left(T_{C C N}\right)_{U H}$ has the universal conservative homomorphism lifting property for finite presentations consists of three parts: (I) the construction of a universal formula $\psi(v)$; (II) a proof that $\left(T_{C C N}\right)_{U H} \cup \operatorname{PDiag}(G) \vdash \chi(\mathbf{a}, \mathbf{b}) \rightarrow \psi(\mathbf{a})$; and (III) a proof that part (ii) in Definition 8 in Appendix A holds. The construction of the formula $\psi$ and the proofs in Parts (II) and (III) depend upon the facts that $G$ has only finitely many distinct homomorphic images and that each finite graph has only finitely many $N$-colorings. 
PART (I). Let $G_{1}, \ldots, G_{q}$ be all the distinct homomorphic images of $G$ which are $N$-colorable and satisfy $\chi\left(\mathbf{a}^{k}, \mathbf{b}^{k}\right)$, respectively, for $k=1, \ldots, q$, where $a_{i}^{k}$ and $b_{j}^{k}$ are the images of $a_{i}$ and $b_{j}$ in $G_{k}$, respectively, for $i=1, \ldots, n, j=1, \ldots, m$. Let $H_{k}$ for $k=1, \ldots, q$ be the subgraph of $G_{k}$ with vertices $a_{1}^{k}, \ldots, a_{n}^{k}$ and no others. Then $H_{k}$ is the image of $H$ under the homomorphism from $G$ onto $G_{k}$.

Define formulas $\varphi_{k}(\mathbf{v})$ and $\psi_{k}(\mathbf{v})$ for $k=1, \ldots, q$ as follows. Let $\varphi_{k}(\mathbf{v})$ be a conjunction of basic formulas such that $\varphi_{k}\left(\mathrm{a}^{k}\right)$ is the conjunction of the sentences in $\operatorname{Diag}\left(H_{k}\right)$. Let $f_{k, 1}, \ldots, f_{k, t(k)}$ be homomorphisms from $H_{k}$ into $K_{N}$ such that (1) none of these homomorphisms can be extended to a homomorphism from $G_{k}$ into $K_{N}$, (2) each homomorphism from $H_{k}$ into $K_{N}$ which cannot be extended to a homomorphism from $G_{k}$ into $K_{N}$ is equivalent to one of $f_{k, 1}, \ldots, f_{k, t(k)}$, and (3) no two of $f_{k, 1}, \ldots, f_{k, t(k)}$ are equivalent. Let $\psi_{k}(\mathbf{v})$ be the formula

$$
\bigwedge_{t=1}^{t(k)} \forall \mathbf{y} \neg\left(\psi\left[H_{k}, f_{k, t}, \mathbf{a}^{k}\right]\right)(\mathbf{v}, \mathbf{y}) .
$$

(If $t(k)=0$, i.e., every homomorphism from $H_{k}$ into $K_{N}$ can be extended to a homomorphism from $G_{k}$ into $K_{N}$, then let $\psi_{k}$ be the empty formula.)

Now let $\psi(v)$ be the formula $\bigvee_{k=1}^{q}\left(\varphi_{k}(v) \wedge \psi_{k}(v)\right)$. It remains to show that $\psi$ satisfies the requirements of Definition 8 in Appendix A relative to $G, H$, and $\chi$.

PART (II). To show that $\left(T_{C C N}\right) U_{H} \cup \operatorname{PDiag}(G)$ implies $\chi(\mathbf{a}, \mathbf{b}) \rightarrow \psi(\mathbf{a})$, suppose that $G^{\prime}$ is a model of $\left(T_{C C N}\right)_{U H} \cup \operatorname{PDiag}(G) \cup\{\chi(\mathbf{a}, \mathbf{b})\}$. Let $a_{1}^{\prime}, \ldots, a_{n}^{\prime}, b_{1}^{\prime}, \ldots, b_{m}^{\prime}$ be the images of $a_{1}, \ldots, a_{n}, b_{1}, \ldots, b_{m}$ under the homomorphism from $G$ into $G^{\prime}$ induced by the interpretation for which $G^{\prime}$ is a model of $\operatorname{PDiag}(G)$. The subgraph $G^{\prime \prime}$ of $G^{\prime}$ whose vertices are $a_{1}^{\prime}, \ldots, a_{n}^{\prime}$, $b_{1}^{\prime}, \ldots, b_{m}^{\prime}$ is isomorphic to one of $G_{1}, \ldots, G_{q}$, because $G^{\prime \prime}$ satisfies $\chi\left(\mathbf{a}^{\prime}, \mathbf{b}^{\prime}\right)$. Suppose that $G^{\prime \prime}$ is isomorphic to $G_{1}$, and let $g$ be the isomorphism from $G_{1}$ onto $G^{\prime \prime}$ defined by $g\left(a_{i}^{1}\right)=a_{i}^{\prime}$ for $i=1, \ldots, n$ and $g\left(b_{j}^{1}\right)=b_{j}^{\prime}$ for $j=$ $1, \ldots, m$. The graph $G^{\prime}$ satisfies $\varphi_{1}\left(\mathbf{a}^{\prime}\right)$, since $g$ maps $H_{1}$ isomorphically onto the subgraph $H^{\prime}$ whose vertices are $a_{1}^{\prime}, \ldots, a_{n}^{\prime}$. Suppose that $G^{\prime}$ does not satisfy $\psi\left(\mathbf{a}^{\prime}\right)$. Then $G^{\prime}$ satisfies $\exists \mathbf{y}\left(\psi\left[H_{1}, f_{1, t}, \mathbf{a}^{1}\right]\right)\left(\mathbf{a}^{\prime}, \mathbf{y}\right)$ for some $t, 1 \leqslant t \leqslant$ $t(1)$; suppose for convenience that this $t$ is 1 . Let $f$ be an $N$-coloring of $G^{\prime}$. Then the restriction of $f \circ g$ to $H_{1}$ is equivalent to $f_{1,1}$, because $G^{\prime}$ satisfies $\exists \mathbf{y}\left(\psi\left[H_{1}, f_{1,1}, \mathbf{a}^{1}\right]\right)\left(\mathbf{a}^{\prime}, \mathbf{y}\right)$. But then $f \circ g$ determines an extension of $f_{1,1}$ to $G_{1}$ contradicting part (1) in the selection of the $f_{k, t}$ 's. Hence, $\left(T_{C C N}\right)_{U H} \cup$ $\operatorname{PDiag}(G) \vdash \chi(\mathbf{a}, \mathbf{b}) \rightarrow \psi(\mathbf{a})$.

PART (III). Suppose now that $H^{\prime \prime}$ is an $N$-colorable graph, $h$ is a homomorphism from $H$ into $H^{\prime \prime}$, and $\psi\left(\mathbf{a}^{\prime \prime}\right)$ is persistable over $H^{\prime \prime}$, where $a_{i}^{\prime \prime}=h\left(a_{i}\right)$ for $i=1, \ldots, n$. Let $H^{\prime \prime \prime}$ be an existentially complete $N$-colorable graph extending $H^{\prime \prime}$ and satisfying $\psi\left(a^{\prime \prime}\right)$. Then $H^{\prime \prime}$ satisfies $\varphi_{k}\left(\mathbf{a}^{\prime \prime}\right) \wedge \psi_{k}\left(\mathbf{a}^{\prime \prime}\right)$ 
for some $k, 1 \leqslant k \leqslant q$; suppose this value of $k$ is 1 . The subgraph $H^{\prime \prime \prime \prime}$ of $H^{\prime \prime}$ whose vertices are $a_{1}^{\prime \prime}, \ldots, a_{n}^{\prime \prime}$ is then isomorphic to $H_{1}$, since $\varphi_{1}\left(\mathbf{a}^{1}\right)$ is the conjunction of the diagram of $H_{1}$. Without loss of generality, one may identify $H_{1}$ and $H^{\prime \prime \prime \prime}$ identifying $a_{i}^{1}$ with $a_{i}^{\prime \prime}$ for $i=1, \ldots, n$. Then the following diagram commutes:

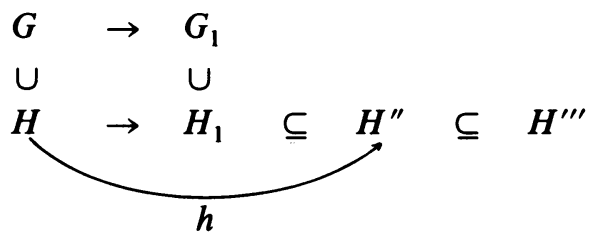

This diagram will be completed by amalgamating $G_{1}$ and $H^{\prime \prime \prime}$ over $H_{1}$. Let $f$ be an $N$-coloring of $H^{\prime \prime \prime}$. The graph $H_{f}^{\prime \prime \prime}$ satisfies $\exists \mathrm{y}\left(\psi\left[H^{\prime \prime \prime}, f, \mathbf{a}^{1}\right]\right)\left(\mathbf{a}^{1}, \mathbf{y}\right)$. Since $H^{\prime \prime \prime}$ is existentially complete, $H^{\prime \prime \prime}$ satisfies $\exists y\left(\psi\left[H^{\prime \prime \prime}, f, \mathbf{a}^{1}\right]\right)\left(\mathbf{a}^{1}, \mathbf{y}\right)$ also. Since $H^{\prime \prime \prime}$ satisfies $\psi_{1}\left(\mathrm{a}^{\mathrm{l}}\right)$, the restriction $f \mid H_{1}$ of $f$ to $H_{1}$ cannot be equivalent to any of $f_{1,1}, \ldots, f_{1, t(1)}$. Therefore, $f \mid H_{1}$ has an extension to a homomorphism from $G_{1}$ into $K_{N}$. According to Lemma 2.1, there exist an $N$-colorable graph $G^{\prime \prime \prime}$ and embeddings $h_{1}$ from $H^{\prime \prime \prime}$ into $G^{\prime \prime \prime}$ and $g_{1}$ from $G_{1}$ into $G^{\prime \prime \prime}$ such that the following diagram commutes:

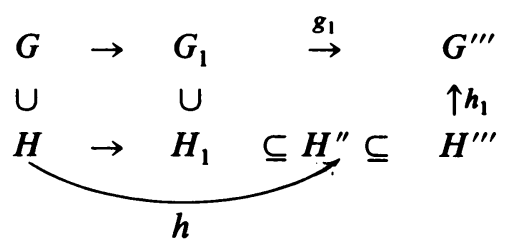

Without loss of generality, $h_{1}$ may be taken to be an inclusion mapping.

Thus, $\left(T_{C C N}\right)_{U H}$ has the universal conservative homomorphism lifting property for finite presentations. Hence, the theory $\left(T_{C C N}\right)_{U H}$ of $N$-colorable graphs has a model-companion.

The model-companion of $\left(T_{C C N}\right)_{U H}$ will be denoted by $\left(T_{C C N}\right)_{U H}^{*}$. This model-companion is not a model-completion for $\left(T_{C C N}\right)_{U H}$, because $\left(T_{C C N}\right)_{U H}$ does not have the amalgamation property.

Proposition 2.3. The theory $\left(T_{C C N}\right)_{U H}^{*}$ is complete.

Proof. The theory $\left(T_{C C N}\right)_{U H}$ has the joint embedding property, and it is well known that the model-companion of a theory with the joint embedding property is complete.

The next proposition answers partially the question, raised by G. Sabbagh, whether the model-companion of a Horn theory, or the model-completion if the theory has the amalgamation property, is necessarily Horn itself.

Proposition 2.4. The theory $\left(T_{C C N}\right)_{U H}^{*}$ is not closed under direct products. 
Proof. Consider again the two vertex graph $H$ with $N$-colorings $f$ and $g$ discussed just prior to the statewment of Theorem 2.2. Let $G_{1}$ and $G_{2}$ be existentially complete $N$-colorable graphs containing $H_{f}$ and $H_{g}$, respectively. Let $G$ be the direct product $G_{1} \times G_{2}$. Let $H^{\prime}$ be the subgraph of $G$ whose vertices are $\left(a_{1}, a_{1}\right)$ and $\left(a_{2}, a_{2}\right)$. For convenience denote by $f$ and $g$ also the homomorphisms from $H^{\prime}$ into $K_{N}$ defined by $f\left(a_{1}^{\prime}\right)=c_{1}, f\left(a_{2}^{\prime}\right)=c_{1}$ and $g\left(a_{1}^{\prime}\right)$ $=c_{1}, g\left(a_{2}^{\prime}\right)=c_{2}$, where $a_{i}^{\prime}$ is $\left(a_{i}, a_{i}\right)$ for $i=1,2$. Since every $N$-coloring of $H^{\prime}$ is equivalent to either $f$ or $g$, each existentially complete $N$-colorable graph containing $H^{\prime}$ satisfies either $\exists \mathbf{y}\left(\psi\left[H^{\prime}, f, \mathbf{a}^{\prime}\right]\right)\left(\mathbf{a}^{\prime}, y\right)$ or $\exists \mathbf{y}\left(\psi\left[H^{\prime}, g, \mathbf{a}^{\prime}\right]\right)\left(\mathbf{a}^{\prime}, \mathbf{y}\right)$. If $H_{g}^{\prime}$ could be embedded over $H^{\prime}$ in $G$, then the projection of $G$ onto $G_{1}$ would yield an embedding of $H_{g}$ over $H$ in $G_{1}$. But no embedding of $H_{g}$ over $H$ in $G_{1}$ can exist, because $H_{f}$ and $H_{g}$ cannot be amalgamated as $N$-colorable graphs over $H$. Therefore $H_{g}^{\prime}$ cannot be embedded over $H^{\prime}$ in $G$. Similarly, $H_{f}^{\prime}$ cannot be embedded over $H^{\prime}$ in $G$. Since neither $H_{f}^{\prime}$ nor $H_{g}^{\prime}$ can be embedded over $H^{\prime}$ into $G, G$ does not satisfy either of the sentences above, so $G$ cannot be existentially complete.

The next goal is to show that $\left(T_{C C N}\right)_{U H}^{*}$ has an effective elimination of quantifiers and is decidable. Of course, the decidability of $\left(T_{C C N}\right)_{U H}^{*}$ could be inferred from Proposition 2.3 if $\left(T_{C C N}\right)_{U H}^{*}$ were recursively axiomatized. However, it is necessary to have the effective elimination of quantifiers in order to obtain this recursive axiomatization.

LEMMA 2.5. There is an algorithm for determining whether a primitive sentence is consistent with $\left(T_{C C N}\right)_{U H}$.

Proof. A suitable algorithm is described as follows. Let $\exists v \theta(v)$ be a primitive sentence where $\theta$ is a conjunction of basic formulas. First one must determine whether $\exists \mathrm{v} \theta$ is consistent with $T_{G R}$. Define a relation $\sim$ on the set $\left\{v_{1}, \ldots, v_{n}\right\}$ by $v_{i} \sim v_{j}$ if and only if $v_{i}=v_{j}$ or $v_{j}=v_{i}$ is a conjunct of $\theta$. Let $\approx$ be the transitive closure of $\sim$, so that $\approx$ is an equivalence relation on $\left\{v_{1}, \ldots, v_{n}\right\}$. The equivalence classes can be effectively determined. If $\theta$ contains a conjunct $\neg\left(v_{i}=v_{j}\right)$ or $E\left(v_{i}, v_{j}\right)$ where $v_{i} \approx v_{j}$, then $\exists \mathrm{v} \theta$ is inconsistent with $T_{G R}$ and so with $\left(T_{C C N}\right)_{U H}$. Suppose then that $\theta$ has no such conjuncts. Let $\boldsymbol{\theta}^{\prime}$ be the formula obtained from $\theta$ by omitting all conjuncts of the form $v_{i}=v_{j}$ and replacing each occurrence of $v_{k}$ by an occurrence of $\operatorname{rep}\left(v_{k}\right)$ where $\operatorname{rep}\left(v_{k}\right)$ is the variable $v_{i}$ for the least integer $i$ for which $v_{k} \approx v_{i}$. After renumbering and changing the value of $n$, one may assume that the variables occurring in $\theta^{\prime}$ are precisely $v_{1}, \ldots, v_{n}$. Furthermore, the original primitive sentence is consistent with $\left(T_{C C N}\right)_{U H}$ if and only if $\exists \mathrm{v} \theta^{\prime}$ is consistent with $\left(T_{C C N}\right)_{U H}$.

Next, determine whether there are indices $i$ and $j, 1 \leqslant i, j \leqslant n$, for which both $E\left(v_{i}, v_{j}\right)$ and either $\neg E\left(v_{i}, v_{j}\right)$ or $\neg E\left(v_{j}, v_{i}\right)$ (or both) are conjuncts of 
$\theta^{\prime}$. If there are such indices, then $\exists \mathrm{v} \theta^{\prime}$ is inconsistent with $T_{G R}$ and so with $\left(T_{C C N}\right)_{U H}$. Suppose then, that there are no such indices.

Let $G$ be the graph with vertices $a_{1}, \ldots, a_{n}$ in which $E\left(a_{i}, a_{j}\right)$ holds if and only if either $E\left(v_{i}, v_{j}\right)$ or $E\left(v_{j}, v_{i}\right)$ occurs in $\theta^{\prime}$. Then $G$ satisfies $\theta^{\prime}(a)$. If $G^{\prime}$ is a graph with vertices $a_{1}^{\prime}, \ldots, a_{n}^{\prime}$ satisfying $\theta^{\prime}\left(\mathbf{a}^{\prime}\right)$, then there is a homomorphism from $G$ into $G^{\prime}$ sending $a_{i}$ to $a_{i}^{\prime}$. Consequently, $\exists v \theta^{\prime}$ is consistent with $\left(T_{C C N}\right)_{U H}$ if and only if $G$ is $N$-colorable. There are at most $N^{n}$ mappings from the vertices of $G$ to vertices of $K_{N}$. These can be effectively listed and determined to be or not be homomorphisms from $G$ into $K_{N}$. Thus, it can be effectively determined whether $G$ is $N$-colorable.

COROLLARY 2.6. The collection of existential sentences which are consequences of $\left(T_{C C N}\right)_{U H}^{*}$ is a decidable set.

Proof. A primitive sentence is a consequence of $\left(T_{C C N}\right)_{U H}^{*}$ if and only if it is consistent with $\left(T_{C C N}\right)_{U H}$, since $\left(T_{C C N}\right)_{U H}$ and $\left(T_{C C N}\right)_{U H}^{*}$ have the same universal consequences and $\left(T_{C C N}\right)_{U H}^{*}$ is complete. Since each existential sentence is equivalent to a disjunction of primitive sentences, which can be effectively constructed, this corollary follows from Lemma 2.5 .

LEMMA 2.7. There is an algorithm for constructing for each primitive formula $\varphi(\mathbf{v})$ a universal formula $\psi(\mathbf{v})$ such $\left(T_{C C N}\right)_{U H}^{*}$ implies $\forall \mathbf{v}(\varphi(\mathbf{v}) \leftrightarrow \psi(\mathbf{v}))$.

Proof. A suitable algorithm is described as follows. Let $\varphi(\mathbf{v})$ be a primitive formula. According to Lemma 2.5 one can effectively determine whether $\exists \mathbf{v} \varphi$ is consistent with $\left(T_{C C N}\right)_{U H}$. If it is not consistent with $\left(T_{C C N}\right)_{U H}$, then let $\psi$ be $\forall x \neg(x=x)$. Suppose then that $\exists \mathrm{v} \varphi$ is consistent with $\left(T_{C C N}\right)_{U H}$. If $\varphi$ is closed, then it is a theorem of $\left(T_{C C N}\right)_{U H}^{*}$, and $\psi$ may be chosen to be $\forall x(x=x)$.

Suppose then that $\varphi$ has at least one free occurrence of a variable. The formula $\varphi$ has the form $\exists x_{1} \cdots \exists x_{m} \theta(\mathbf{v}, \mathbf{x})$ where $\theta$ is a conjunction of basic formulas. As in the proof of Lemma 2.5 , define a relation $\sim$ on $\left\{v_{1}, \ldots, v_{n}\right.$, $\left.x_{1}, \ldots, x_{m}\right\}=V$ by $w \sim z$ for elements $w$ and $z$ of $V$ if and only if $w=z$ or $z=w$ is a conjunct of $\theta$. Let $\approx$ be the transitive closure of $\sim$, so that $\approx$ is an equivalence relation on $V$ and the equivalence classes can be effectively determined. For each variable $w$ in $V$ choose a representative $\operatorname{rep}(w)$ for the equivalence class of $w$ as follows: (i) if $w \approx v_{j}$ for some $j$, then let $\operatorname{rep}(w)=v_{i}$ where $i$ is the least integer for which $w \approx v_{i}$; (ii) otherwise, let $\operatorname{rep}(w)=x_{i}$ where $i$ is the least integer for which $w \approx x_{i}$. Now let $\rho(v)$ be the conjunction $\bigwedge_{j=1}^{n} v_{j}=\operatorname{rep}\left(v_{j}\right)$. Let $\sigma(\mathbf{v}, \mathbf{x})$ be the conjunction $\bigwedge_{j=1}^{m} x_{j}=\operatorname{rep}\left(x_{j}\right)$. Let $\theta^{\prime}(\mathbf{v}, \mathbf{x})$ be the conjunction of all the formulas $\neg(\operatorname{rep}(w)=\operatorname{rep}(z))$, $E(\operatorname{rep}(w), \operatorname{rep}(z))$, and $\neg E(\operatorname{rep}(w), \operatorname{rep}(z))$ for which $\neg(w=z), E(w, z)$, and $\neg E(w, z)$, respectively, are conjuncts of $\theta$, where $w$ and $z$ are variables in $V$. Then $\theta$ is logically equivalent to $\rho \wedge \sigma \wedge \theta^{\prime}$ and $\exists \mathrm{x} \theta$ is logically 
equivalent to $\rho \wedge \exists x \theta^{\prime}$. After renumbering the variables one may assume that the variables occurring in $\theta^{\prime}$ are precisely $v_{1}, \ldots, v_{p}, x_{1}, \ldots, x_{q}$ where $1 \leqslant p \leqslant n, 0 \leqslant q \leqslant m$.

Let $G$ be the graph with vertices $a_{1}, \ldots, a_{p}, b_{1}, \ldots, b_{q}$ in which $E\left(a_{i}, a_{j}\right)$, $E\left(a_{i}, b_{j}\right)$, and $E\left(b_{i}, b_{j}\right)$ hold if and only if $E\left(v_{i}, v_{j}\right)$ or $E\left(v_{j}, v_{i}\right), E\left(v_{i}, x_{j}\right)$ or $E\left(x_{j}, v_{i}\right)$, and $E\left(x_{i}, x_{j}\right)$ or $E\left(x_{j}, x_{i}\right)$, respectively, are conjuncts of $\theta^{\prime}$. Then $G$ satisfies $\theta^{\prime}\left(a_{1}, \ldots, a_{p}, b_{1}, \ldots, b_{q}\right)$. Moreover, if $G^{\prime}$ is a graph with vertices $a_{1}^{\prime}, \ldots, a_{p}^{\prime}, b_{1}^{\prime}, \ldots, b_{q}^{\prime}$ satisfying $\theta^{\prime}\left(a_{1}^{\prime}, \ldots, a_{p}^{\prime}, b_{1}^{\prime}, \ldots, b_{q}^{\prime}\right)$, then there is a homomorphism from $G$ into $G^{\prime}$ sending $a_{i}$ to $a_{i}^{\prime}$ and $b_{j}$ to $b_{j}^{\prime}$ for $i=1, \ldots, p$, $j=1, \ldots, q$. Let $H$ be the subgraph of $G$ whose vertices are $a_{1}, \ldots, a_{p}$. Let $\chi\left(v_{1}, \ldots, v_{p}, x_{1}, \ldots, x_{q}\right)$ be the conjunction of the negated atomic conjuncts of $\theta^{\prime}$. According to the proof of Theorem 1 of [9], $\left(T_{C C N}\right)_{U H}^{*}$ contains a sentence $\forall v_{1} \cdots \forall v_{p}\left[\left(\exists x_{1} \cdots \exists x_{q} \theta^{\prime}\right) \leftrightarrow\left(\theta_{1}\left(v_{1}, \ldots, v_{p}\right) \wedge \psi_{1}\left(v_{1}, \ldots, v_{p}\right)\right)\right]$ where $\theta_{1}$ is a presentation of $H$ and $\psi_{1}$ is the formula $\psi$ constructed in the proof of Theorem 2.2 for this $G, H$, and $\chi$. Clearly, $\theta_{1}$ may be chosen to be the conjunction of the conjuncts $E\left(v_{i}, v_{j}\right)$ of $\theta^{\prime}$, so $\theta_{1}$ has been effectively constructed. The construction of $\psi$ in the proof of Theorem 2.2 can be performed effectively, because $G$ has only finitely many distinct homomorphic images, which can be effectively listed, and each finite graph has only finitely many mappings into $K_{N}$. Thus, the universal formula $\rho \wedge \theta_{1} \wedge \psi_{1}$ can be effectively constructed, and $\left(T_{C C N}\right)_{U H}^{*}$ implies $\forall \mathbf{v}(\exists \mathbf{x} \theta \leftrightarrow$ $\left.\left(\rho \wedge \theta_{1} \wedge \psi_{1}\right)\right)$.

THEOREM 2.8. There is an algorithm for constructing for each formula $\Phi(\mathbf{v})$ an existential formula $\varphi(\mathbf{v})$ such that $\left(T_{C C N}\right)_{U H}^{*}$ implies $\forall \mathbf{v}(\Phi \leftrightarrow \varphi)$.

Proof. It suffices to consider formulas $\Phi$ in prenex form. If $\Phi$ is existential, then let $\varphi$ be $\Phi$. If $\Phi$ is universal, then $\neg \Phi$ into the form of a disjunction of primitive formulas $\Phi_{1} \vee \cdots \vee \Phi_{k}$. According to Lemma 2.7 one can effectively construct universal formulas $\psi_{1}, \ldots, \psi_{k}$ such that $\left(T_{C C N}\right)_{U H}^{*}$ implies $\forall \mathrm{v}\left[\Phi_{i} \leftrightarrow \psi_{i}\right]$ for $i=1, \ldots, k$. Let $\varphi$ be $\neg\left(\psi_{1} \vee \cdots \vee \psi_{k}\right)$.

Assume then that the theorem is true for formulas with at most $m$ blocks of quantifiers. Suppose $\Phi$ is $\exists \mathbf{x} \Phi_{1}$ where $\Phi_{1}$ is $\forall_{m}$. By induction there is an effectively constructed existential formula $\varphi_{1}(\mathbf{v}, \mathbf{x})$ for which $\left(T_{C C N}\right)_{U H}^{*}$ implies $\forall \mathbf{v} \forall \mathbf{x}\left(\Phi_{1} \leftrightarrow \varphi_{1}\right)$. Then $\left(T_{C C N}\right)_{U H}^{*}$ implies $\forall \mathbf{v}\left(\Phi \leftrightarrow \exists \mathbf{x} \varphi_{1}\right)$. Suppose on the other hand that $\Phi$ is $\forall \mathbf{x} \Phi_{1}$ where $\Phi_{1}$ is $\exists_{m}$. By induction there is an effectively constructed existential formula $\varphi_{2}(\mathbf{v}, \mathbf{x})$ for which $\left(T_{C C N}\right)_{U H}^{*}$ implies $\forall \mathbf{v} \forall \mathbf{x}\left(\neg \Phi_{1} \leftrightarrow \varphi_{2}\right)$. According to the case of universal formulas, there is an effectively constructed existential formula $\varphi_{1}(v, w)$ for which $\left(T_{C C N}\right)_{U H}^{*}$ implies $\forall \mathrm{v}\left[\left(\forall \mathbf{x} \neg \varphi_{2}\right) \leftrightarrow \varphi_{1}\right]$. Then $\left(T_{C C N}\right)_{U H}^{*}$ implies $\forall \mathbf{v}\left(\Phi \leftrightarrow \varphi_{1}\right)$.

THEOREM 2.9. The theory $\left(T_{C C N}\right)_{U H}^{*}$ is decidable. 
Proof. Let $\Phi$ be a sentence. According to Theorem 2.8, there is an effectively constructed, existential sentence $\varphi$ such that $\left(T_{C C N}\right)_{U H}^{*}$ implies $\Phi \leftrightarrow \varphi$. Whether $\varphi$ is a theorem of $\left(T_{C C N}\right)_{U H}^{*}$ can be effectively determined according to Corollary 2.6 .

The next goal is to show that $\left(T_{C C N}\right)_{U H}^{*}$ is $\aleph_{0}$-categorical and independent.

An $n$-type $\Delta$ for $\left(T_{C C N}\right)_{U H}$ will be a collection of formulas with free variables among $v_{1}, \ldots, v_{n}$ which are simultaneously realized by elements $a_{1}, \ldots, a_{n}$ of some model of $\left(T_{C C N}\right)_{U H}$. An $n$-type $\Delta$ is complete if, for each formula $\varphi(v)$, either $\varphi$ or $\neg \varphi$ is in $\Delta$. An $n$-type $\Delta$ is an existential $n$-type if each formula in $\Delta$ is existential. An existential $n$-type $\Delta$ is a maximal existential $n$-type for $\left(T_{C C N}\right)_{U H}$ if, for each existential formula $\varphi(v)$, either $\varphi$ is in $\Delta$ or $\left(T_{C C N}\right)_{U H} \cup \Delta$ implies $\neg \varphi$.

LEMMA 2.10. Each maximal existential n-type for $\left(T_{C C N}\right)_{U H}$ is uniquely determined by the diagram of an $N$-colorable graph $G$ with vertices $a_{1}, \ldots, a_{n}$ only (not necessarily distinct) and the formula $\exists \mathrm{v}(\psi[G, f, \mathbf{a}])(\mathbf{v}, \mathbf{y})$ for an $N$ coloring $f$ of $G$.

Proof. Let $\Delta$ be a maximal existential $n$-type for $\left(T_{C C N}\right)_{U H}$. If $\rho(v)$ is an atomic formula, then either $\rho$ is in $\Delta$ or $\left(T_{C C N}\right)_{U H} \cup \Delta$ implies $\neg \rho$, in which case $\neg \rho$ must be in $\Delta$ since $\left(T_{C C N}\right)_{U H} \cup \Delta$ cannot then imply $\neg \neg \rho$ as well. Let $\theta(\mathbf{v})$ be the conjunction of all the basic formulas in $\Delta$. There is a graph $G$ with vertices $a_{1}, \ldots, a_{n}$ (not necessarily distinct) and no other vertices for which $\theta(a)$ is the conjunction of the diagram of $G$.

Suppose that $G^{\prime}$ is an $N$-colorable graph containing vertices $a_{1}^{\prime}, \ldots, a_{n}^{\prime}$ which realize $\Delta$. Then the subgraph whose vertices are $a_{1}^{\prime}, \ldots, a_{n}^{\prime}$ is naturally isomorphic to $G$, and so $a_{i}$ can be identified with $a_{i}^{\prime}$ for $i=1, \ldots, n$. Since $G^{\prime}$ is $N$-colorable, there is a homomorphism from $G^{\prime}$ into $K_{N}$. Then the graph $G_{f}^{\prime}$ satisfies $\exists \mathbf{y}(\psi[G, f \mid G, \mathbf{a}])(\mathbf{a}, \mathbf{y})$, where $f \mid G$ is the restriction of $f$ to $G$. Therefore $\exists \mathrm{y}(\psi[G, f \mid G, \mathbf{a}])(\mathbf{v}, \mathbf{y})$ is consistent with $\left(T_{C C N}\right)_{U H} \cup \Delta$ and so is in $\Delta$.

Let $\varphi(v)$ be an existential formula. If $\varphi$ is in $\Delta$, then $\varphi$ is consistent with $\left(T_{C C N}\right)_{U H} \cup\{\theta \wedge \exists \mathbf{y}(\psi[G, f \mid G, \mathbf{a}])(\mathbf{v}, \mathbf{y})\}$. Conversely, suppose that $\varphi$ is consistent with $\left(T_{C C N}\right)_{U H} \cup\{\theta \wedge \exists \mathbf{y}(\psi[G, f \mid G, \mathbf{a}])(\mathbf{v}, \mathbf{y})\}$. Then there is an $N$-colorable graph $G^{\prime \prime}$ extending $G$ which satisfies $\varphi($ a) and which has an $N$-coloring which coincides with $f \mid G$ on $G$. Consequently, $G^{\prime}$ and $G^{\prime \prime}$ can be amalgamated over $G$ as $N$-colorable graphs. Therefore, $\varphi$ is consistent with $\left(T_{C C N}\right)_{U H} \cup \Delta$, and so $\varphi$ is in $\Delta$. Thus, $\varphi$ is in $\Delta$ if and only if $\varphi$ is consistent with $\left(T_{C C N}\right)_{U H} \cup\{\theta \wedge \exists y(\psi[G, f \mid G, \mathbf{a}])(\mathbf{v}, \mathbf{y})\}$.

PROPOSITION 2.11. The theory $\left(T_{C C N}\right)_{U H}$ has only finitely many maximal existential $n$-types for each $n$. 
Proof. This follows from Lemma 2.10 , because there are only finitely many $N$-colorable graphs with at most $n$ vertices for each $n$ and each of these graphs has only finitely many $N$-colorings.

TheOREM 2.12. The theory $\left(T_{C C N}\right)_{U H}^{*}$ is $\aleph_{0}$-categorical.

Proof. Each complete $n$-type $\Delta^{\prime}$ for $\left(T_{C C N}\right)_{U H}^{*}$ is uniquely determined by the subset $\Delta$ of all existential formulas in $\Delta^{\prime}$, because $\left(T_{C C N}\right)_{U H}^{*}$ is modelcomplete. Each model of $\left(T_{C C N}\right)_{U H}^{*}$ is existentially complete, so according to Proposition 1.28 of [3], each sequence of $n$ elements in a model of $\left(T_{C C N}\right)_{U H}^{*}$ must realize a maximal existential $n$-type for $\left(T_{C C N}\right)_{U H}$. Therefore the subtype $\Delta$ of $\Delta^{\prime}$ must be a maximal existential $n$-type for $\left(T_{C C N}\right)_{U H}$. Since $\left(T_{C C N}\right)_{U H}$ has only finitely many maximal existential $n$-types for each $n,\left(T_{C C N}\right)_{U H}^{*}$ has only finitely many complete types for each $n$. Therefore $\left(T_{C C N}\right)_{U H}^{*}$ is $\aleph_{0}$-categorical by the Ryll-Nardjewski Theorem [8].

The existence of $\left(T_{C C N}\right)_{U H}^{*}$ could have been inferred from Theorem 6.4 of [3] using Proposition 2.12. However, the decidability of $\left(T_{C C N}\right)_{U H}^{*}$ and its effective elimination of quantifiers could not then be deduced except by duplicating the proofs of Theorem 2.2 through Theorem 2.9.

LeMmA 2.13. Let $G$ be a graph with an $N$-coloring $f$. Let $\left\{a_{\alpha}: \alpha \in A\right\}$ be a set of vertices of $G$ for which $f\left(a_{\alpha}\right)=f\left(a_{\beta}\right)$ for all $\alpha$ and $\beta$ in $A$. Then for each subset $A^{\prime}$ of $A$ the set of sentences $\left(T_{C C N}\right)_{U H} \cup \operatorname{Diag}(G) \cup\left\{E\left(a_{\alpha}, b\right): \alpha \in A^{\prime}\right\}$ $\cup\left\{\neg E\left(a_{\alpha}, b\right) ; \alpha \in A-A^{\prime}\right\}$, where $b$ is a constant symbol not occurring in the language of $G$, is consistent.

Proof. Let $G\left(A^{\prime}\right)$ be the graph whose set of vertices consists of the vertices of $G$ and a new vertex $b$ and in which two vertices $c$ and $d$ are adjacent if and only if (i) $c$ and $d$ are vertices of $G$ and are adjacent in $G$, or (ii) $c$ is $b$ and $d$ is $a_{\alpha}$ for some $\alpha$ in $A^{\prime}$ or vice-versa. One may assume after replacing $f$ by a suitable equivalent $N$-coloring that $f\left(a_{\alpha}\right)=c_{1}$ for all $\alpha$ in $A$. Define a mapping $f^{\prime}$ from $G\left(A^{\prime}\right)$ into $K_{N}$ by $f^{\prime}(x)=f(x)$ for all $x$ in $G$ and $f^{\prime}(b)=c_{2}$. Then $f$ is a homomorphism, so $G\left(A^{\prime}\right)$ is $N$-colorable.

The reader is referred to [7] for definitions of independence for complete theories and formulas in their languages and to [4] for definitions of independence for universal theories and conjunctions of basic formulas in their languages.

THEOREM 2.14. The formula $E\left(v_{1}, x_{1}\right)$ is independent for $\left(T_{C C N}\right)_{U H}$ and $\left(T_{C C N}\right)_{U H}^{*}$

Proof. Let $G$ be a denumerably infinite, $N$-colorable graph with an $N$-coloring $f$. Then there is a set $\left\{a_{\alpha}: \alpha<\omega\right\}$ of vertices of $G$ for which 
$f\left(a_{\alpha}\right)=f\left(a_{\beta}\right)$ for all $\alpha, \beta<\omega$. For each subset $A^{\prime}$ of $\omega$ the type $\Delta\left(A^{\prime}\right)=$ $\left\{E\left(a_{\alpha}, x_{1}\right): \alpha \in A^{\prime}\right\} \cup\left\{\neg E\left(a_{\alpha}, x_{1}\right): \alpha \in \omega-A^{\prime}\right\}$ is consistent with $T \cup$ $\operatorname{Diag}\left(G_{f}\right)$ according to the preceding lemma. So, $E$ is independent for $\left(T_{C C N}\right)_{U H}$. Since $G_{f}$ is an amalgamation base, there is an $N$-colorable graph $G^{\prime}$ extending $G_{f}$ which realizes $\Delta\left(A^{\prime}\right)$ for each subset $A^{\prime}$ of $\omega$. Since $G^{\prime}$ can be embedded in a model of $\left(T_{C C N}\right)_{U H}^{*}, E$ is independent for $\left(T_{C C N}\right)_{U H}^{*}$.

CoROllary 2.15. The theories $\left(T_{C C N}\right)_{U H}$ and $\left(T_{C C N}\right)_{U H}^{*}$ are independent.

The final goal of this section is the characterization of the $N$-colorable graphs which have prime model extensions for the theory $\left(T_{C C N}\right)_{U H}^{*}$.

If $G$ is an $N$-colorable graph and $G^{\prime}$ is an $N$-colorable graph extending $G$, then $G^{\prime}$ will be called an $N$-colorable extension of $G$.

Definition. A sequence consisting of an $N$-colorable graph $G$, an infinite index set $A$, a set $\left\{d_{\alpha}: \alpha \in A\right\}$ of vertices of $G$ all of which are assigned the same color under every $N$-coloring of $G$ and which satisfy $d_{\alpha} \neq d_{\beta}$ for $\alpha \neq \beta$, a nonempty subset $A^{\prime}$ of $A$, a primitive formula $\varphi\left(v_{1}, \mathbf{x}\right)$, vertices $b_{1}, \ldots, b_{m}$ of $G$, and a 1-type $\Delta=\left\{\varphi\left(v_{1}, \mathbf{b}\right)\right\} \cup\left\{E\left(v_{1}, d_{\alpha}\right): \alpha \in A^{\prime}\right\} \cup\left\{\neg E\left(v_{1}, d_{\alpha}\right)\right.$ : $\left.\alpha \in A-A^{\prime}\right\}$ which is not realized by any vertex of $G$ in any $N$-colorable extension of $G$ will be called an omission configuration for $\left(T_{C C N}\right)_{U H}$.

Lemma 2.16. Assume that the sequence $G, A,\left\{d_{\alpha}: \alpha \in A\right\}, A^{\prime}, \varphi\left(v_{1}, \mathbf{x}\right)$, $b_{1}, \ldots, b_{m}, \Delta$ is an omission configuration for $\left(T_{C C N}\right)_{U H}$. If $\theta(\mathbf{v}, \mathbf{x})$ is a conjunction of basic formulas in the language of $T_{G R}$, no conjunct of $\theta$ has the form $w=z$ where $w$ and $z$ are variables, and $\exists \mathbf{v} \theta(\mathbf{v}, \mathbf{b})$ is satisfied by some $N$-colorable extension of $G$, then there is an $N$-colorable extension $G^{\prime}$ of $G$ with elements $a_{1}, \ldots, a_{n}$ such that $G^{\prime}$ satisfies $\theta(\mathbf{a}, \mathbf{b})$ and the sequence $G^{\prime}, A,\left\{d_{\alpha}\right.$ : $\alpha \in A\}, A^{\prime}, \varphi^{\prime}, b_{1}, \ldots, b_{m}, \Delta$ is an omission configuration for $\left(T_{C C N}\right)_{U H}$.

Proof. There are two cases.

Case I. There is an $N$-colorable graph $G^{\prime}$ extending $G$ such that $G^{\prime}$ satisfies $\exists \mathrm{v} \theta(\mathbf{v}, \mathbf{b})$ and each element of $G^{\prime}$ which satisfies $\varphi\left(v_{1}, \mathbf{b}\right)$ in an $N$-colorable extension of $G^{\prime}$ either is in $G$ itself or is not adjacent to any $d_{\alpha}$ with $\alpha \in A^{\prime}$. Then $G^{\prime}$ satisfies the conclusion of the lemma, since, for the first alternative, no element of $G$ can realize $\Delta$ in an $N$-colorable extension of $G^{\prime}$ and, for the second alternative, $A^{\prime} \neq \varnothing$.

Case II. Assume that each $N$-colorable graph extending $G$ and satisfying $\exists \mathrm{v} \theta(\mathbf{v}, \mathbf{b})$ contains a vertex $c$ which is not in $G$, satisfies $\varphi(c, \mathbf{b})$ in some further $N$-colorable extension, and is adjacent to an element $d_{\alpha}$ with $\alpha \in A^{\prime}$.

Let $G^{\prime \prime}$ be the graph whose set of vertices consists of the vertices of $G$ and new vertices $a_{1}, \ldots, a_{n}$ and in which two vertices $c$ and $d$ are adjacent if and only if (1) $c$ and $d$ are in $G$ and are adjacent in $G$ or (2) $E(c, d)$ or $E(d, c)$ is a conjunct of $\theta(\mathbf{a}, \mathbf{b})$. Then $G^{\prime \prime}$ satisfies $\theta(\mathbf{a}, \mathbf{b})$. Moreover, if $G^{\prime \prime \prime}$ is an 
$N$-colorable extension of $G$ and satisfies $\theta\left(\mathbf{a}^{\prime}, \mathbf{b}\right)$ for some vertices $a_{1}^{\prime}, \ldots, a_{n}^{\prime}$, then there is a homomorphism from $G^{\prime \prime}$ into $G^{\prime \prime \prime}$ which is the identity on $G$ and maps $a_{i}$ to $a_{i}^{\prime}$ for $i=1, \ldots, n$. Since $\exists v \theta(\mathbf{v}, \mathbf{b})$ is satisfied in some $N$-colorable extension of $G$ by hypothesis, the graph $G^{\prime \prime}$ is $N$-colorable.

Construct a graph $G^{\prime}$ from $G^{\prime \prime}$ as follows. Since $\left\{d_{\alpha}: \alpha \in A\right\}$ is infinite, there is a $\beta$ in $A$ for which $d_{\beta}$ is unequal to any of $a_{1}, \ldots, a_{n}, b_{1}, \ldots, b_{m}$. Consequently, $G^{\prime \prime}$ satisfies $\neg E\left(a_{i}, d_{\beta}\right)$ for $i=1, \ldots, n$. If $\beta$ is in $A^{\prime}$, then let $G^{\prime}$ be $G^{\prime \prime}$. If $\beta$ is not in $A^{\prime}$, then let $G^{\prime}$ be the graph obtained by adding to $G^{\prime \prime}$ an edge $E\left(a_{i}, d_{\beta}\right)$ for each $i, 1 \leqslant i \leqslant n$, such that $a_{i}$ is adjacent in $G^{\prime \prime}$ to $d_{\alpha}$ for some $\alpha \in A$. If $f$ is an $N$-coloring for $G^{\prime \prime}$, then $f$ is an $N$-coloring for $G^{\prime}$ also, because each $a_{i}$ for which an edge $E\left(a_{i}, d_{\beta}\right)$ was added was adjacent in $G^{\prime \prime}$ to some $d_{\alpha}$ so that $f\left(d_{\beta}\right)=f\left(d_{\alpha}\right) \neq f\left(a_{i}\right)$. Therefore $G^{\prime}$ is an $N$-colorable graph extending $G$. The graph $G^{\prime}$ satisfies $\theta(\mathbf{a}, \mathbf{b})$, because $G^{\prime \prime}$ satisfied $\boldsymbol{\theta}(\mathbf{a}, \mathbf{b})$ and $d_{\beta}$ was distinct from $a_{1}, \ldots, a_{n}, b_{1}, \ldots, b_{m}$. Finally, none of $a_{1}, \ldots, a_{n}$ can realize $\Delta$ in any $N$-colorable extension of $G^{\prime}$, for each $a_{i}$ either is not adjacent to $d_{\beta}$ for some $\beta$ in $A^{\prime}$ or is adjacent to $d_{\beta}$ for some $\beta$ in $A-A^{\prime}$. Thus, $G^{\prime}$ satisfies the conclusion of the lemma.

LEMMA 2.17. If the sequence $G, A,\left\{d_{\alpha}: \alpha \in A\right\}, A^{\prime}, \varphi\left(v_{1}, \mathbf{x}\right), b_{1}, \ldots, b_{m}, \Delta$ is an omission configuration for $\left(T_{C C N}\right)_{U H}$, then there is an $N$-colorable extension $G^{*}$ of $G$ such that (i) the sequence $G^{*}, A,\left\{d_{\alpha}: \alpha \in A\right\}, A^{\prime}, \varphi\left(v_{1}, \mathbf{x}\right)$, $b_{1}, \ldots, b_{m}, \Delta$ is an omission configuration for $\left(T_{C C N}\right)_{U H}$ and (ii) each primitive formula defined in $G$ and realized in some $N$-colorable extension of $G^{*}$ is realized in $G^{*}$ itself.

Proof. Let $\left\{\boldsymbol{\theta}_{\gamma}\left(\mathbf{v}, \mathbf{b}^{\gamma}\right): \gamma<\kappa\right\}$, where $\kappa=\boldsymbol{\aleph}_{0}+\operatorname{card}(G)$, be an enumeration of all the conjunctions of basic formulas defined in $G$ such that no conjunct has the form $w=z$ where $w$ and $z$ are variables or constants. Let $G_{0}=G$. Construct inductively an ascending chain $G_{0} \subseteq G_{1} \subseteq \ldots$ of $N$ colorable graphs such that the sequence $G_{\gamma}, A,\left\{d_{\alpha}: \alpha \in A\right\}, A^{\prime}, \varphi\left(v_{1}, \mathbf{x}\right)$, $b_{1}, \ldots, b_{m}, \Delta$ is an omission configuration for $\left(T_{C C N}\right)_{U H}$ for each $\gamma<\kappa$ as follows:

(i) $\gamma=\beta+1$. If $\exists v \theta_{\beta}\left(\mathbf{v}, \mathbf{b}^{\beta}\right)$ is satisfied in an $N$-colorable extension of $G_{\beta}$, then according to Lemma 2.16 (after a suitable increase in the value of $\boldsymbol{m}$ and a renumbering of $\left.b_{1}, \ldots, b_{m} b_{1}^{\beta}, \ldots, b_{m(\beta)}^{\beta}\right)$ there is an $N$-colorable extension $G_{\beta+1}$ of $G_{\beta}$ such that $G_{\beta+1}$ satisfies $\exists v \theta_{\beta}\left(\mathbf{v}, \mathbf{b}^{\beta}\right)$ and the sequence $G_{\beta+1}, A$, $\left\{d_{\alpha}: \alpha \in A\right\}, A^{\prime}, \varphi\left(v_{1}, \mathbf{x}\right), b_{1}, \ldots, b_{m}, \Delta$ is an omission configuration for $\left(T_{C C N}\right)_{U H}$. Otherwise, if $\exists v \theta_{\beta}\left(\mathbf{v}, \mathbf{b}^{\beta}\right)$ is not satisfied in any $N$-colorable extension of $G_{\beta}$, then let $G_{\beta+1}=G_{\beta}$.

(ii) $\gamma$ is a limit ordinal. Then let $G_{\gamma}=\cup_{\beta<\gamma} G_{\beta}$. Then the sequence $G_{\gamma}, A$, $\left\{d_{\alpha}: \alpha \in A\right\}, A^{\prime}, \varphi\left(v_{1}, \mathbf{x}\right), b_{1}, \ldots, b_{m}, \Delta$ is an omission configuration for $\left(T_{C C N}\right)_{U H}$. 
Now let $G^{*}=\cup_{\gamma<\kappa} G_{\gamma}$. Clearly, $G^{*}$ satisfies the conclusion of this lemma.

LEMMA 2.18. If the sequence $G_{\gamma}, A,\left\{d_{\alpha}: \alpha \in A\right\}, A^{\prime}, \varphi\left(v_{1}, \mathbf{x}\right) b_{1}, \ldots, b_{m}, \Delta$ is an omission configuration for $\left(T_{C C N}\right)_{U H}$, then there is an existentially complete $N$-colorable graph $\bar{G}$ extending $G$ such that no element of $\bar{G}$ realizes $\Delta$.

Proof. Construct an ascending chain of $N$-colorable graphs inductively as follows. Let $G^{0}=G$. For each $n \geqslant 0$, let $G^{n+1}=\left(G^{n}\right)^{*}$ be the graph obtained by applying Lemma 2.17 to $G^{n}$. Now let $G^{\omega}=\cup_{n<\omega} G^{n}$. Then $G^{\omega}$ is an existentially complete $N$-colorable graph, and no element of $G^{\omega}$ realizes $\Delta$.

THEOREM 2.19. An $N$-colorable graph which is not a model of $\left(T_{C C N}\right)_{U H}^{*}$ has a prime model extension for $\left(T_{C C N}\right)_{U H}^{*}$ if and only if it is finite and an amalgamation base.

Proof. Sufficiency. Suppose that an $N$-colorable graph $G$ is finite and an amalgamation base. Then $\left(T_{C C N}\right)_{U H} \cup \operatorname{Diag}(G)$ has the joint embedding property. Now $\left(T_{C C N}\right)_{U H}^{*} \cup \operatorname{Diag}(G)$ is a model-companion for $\left(T_{C C N}\right)_{U H} \cup$ $\operatorname{Diag}(G)$ and so must be complete. Each maximal existential $n$-type for $\left(T_{C C N}\right)_{U H} \cup \operatorname{Diag}(G)$ is uniquely determined by the diagram of an $N$ colorable graph which extends $G$ and has at most $n$ vertices distinct from the vertices of $G$ and an $N$-coloring of this $N$-colorable extension of $G$. Consequently, $\left(T_{C C N}\right)_{U H}^{*} \cup \operatorname{Diag}(G)$ has only finitely many complete $n$-types for $n$, so $\left(T_{C C N}\right)_{U H}^{*} \cup \operatorname{Diag}(G)$ is $\aleph_{0}$-categorical. The models of $\left(T_{C C N}\right)_{U H}^{*} \cup$ $\operatorname{Diag}(G)$ are just the models of $\left(T_{C C N}\right)_{U H}^{*}$ which have a distinguished subgraph isomorphic to $G$. Thus, the unique countable model of $\left(T_{C C N}\right)_{U H}^{*}$ is a prime model extension of $G$ for $\left(T_{C C N}\right)_{U H}^{*}$.

Necessity. Suppose that an $N$-colorable graph $G$ which is not a model of $\left(T_{C C N}\right)_{U H}^{*}$ has a prime model extension for $\left(T_{C C N}\right)_{U H}^{*}$. Then $\left(T_{C C N}\right)_{U H}^{*} \cup$ $\operatorname{Diag}(G)$, the model-companion of $\left(T_{C C N}\right)_{U H} \cup \operatorname{Diag}(G)$, is a complete theory; so, $\left(T_{C C N}\right)_{U H} \cup \operatorname{Diag}(G)$ has the joint embedding property, or equivalently $G$ is an amalgamation base.

Suppose further that $G$ is infinite. The graph $G$ has a unique $N$-coloring up to equivalence, since $G$ is an amalgamation base. Therefore, since $G$ is infinite, there is an infinite set of vertices $\left\{d_{\alpha}: \alpha \in A\right\}$ of $G$ all of which are assigned the same color by each $N$-coloring of $G$ and which satisfy $d_{\alpha} \neq d_{\beta}$ for $\alpha \neq \beta$. Let $f$ be an $N$-coloring of $G$. There are two cases to be considered.

Case I. Suppose there is a vertex of $K_{N}$ whose inverse image under $f$ is finite, consisting of vertices $b_{1}, \ldots, b_{m}$ and no others $(m=0$ is permitted, i.e., the inverse image is the empty set). Let $e_{1}, \ldots, e_{p}$ be vertices of $G$ such that $f\left(e_{i}\right) \neq f\left(e_{j}\right)$ for $i \neq j, f\left(e_{i}\right) \neq f\left(b_{1}\right)$ for $i=1, \ldots, p$, and for each vertex $c$ of $G$ distinct from $b_{1}, \ldots, b_{m} f(c)=f\left(e_{i}\right)$ for some $i, 1<i<p$. Let 
$\varphi_{1}\left(v_{1}, \mathrm{~b}, \mathbf{e}\right)$ be the formula

$$
\begin{aligned}
\exists z_{1} \cdots \exists z_{N}\left[\bigwedge_{i=1}^{N-1} \bigwedge_{j=i+1}^{N} E\left(z_{i}, z_{j}\right)\right. & \\
\wedge \bigwedge_{i=1}^{p} \bigwedge_{j=1, i \neq j}^{N} E\left(e_{i}, z_{j}\right) \wedge \bigwedge_{i=1}^{m} \bigwedge_{j=1, j \neq p+1}^{N} E\left(b_{i}, z_{j}\right) & \left.\bigwedge_{i=1, j \neq p+1}^{N} E\left(v_{1}, z_{j}\right) \wedge \bigwedge_{i=1}^{m} \neg\left(v_{1}=b_{i}\right)\right]
\end{aligned}
$$

where the obvious changes are made if $m=0$. Let $\beta$ be an element of $A$. Let $\varphi\left(v_{1}, \mathbf{b}, d_{\beta}, \mathbf{e}\right)$ be $\varphi\left(v_{1}, \mathbf{b}, \mathbf{e}\right) \wedge E\left(v_{1}, d_{\beta}\right)$. No element of $G$ can satisfy $\varphi\left(v_{1}, \mathbf{b}, d_{\beta}, \mathbf{e}\right)$ in any $N$-colorable extension of $G$, but $\exists v_{1} \varphi\left(v_{1}, \mathbf{b}, d_{\beta}, \mathbf{e}\right)$ is satisfied in some $N$-colorable extension of $G$.

Let $G^{\prime}$ be an existentially complete $N$-colorable graph extending $G$. Then $G^{\prime}$ satisfies $\exists v_{1} \varphi\left(v_{1}, \mathbf{b}, d_{\beta}, \mathbf{e}\right)$, since $G$ is an amalgamation base and this sentence is satisfied in some $N$-colorable extension of $G$. Let $c$ be a vertex of $G^{\prime}$ for which $G^{\prime}$ satisfies $\varphi\left(c, \mathbf{b}, d_{\beta}, \mathbf{e}\right)$. Let $A^{\prime}=\left\{\alpha: \alpha \in A\right.$ and $G^{\prime}$ satisfies $\left.E\left(c, d_{\alpha}\right)\right\}$. Then $\beta \in A^{\prime}$, so $A^{\prime}$ is not empty. According to Lemma 2.18 , there is an existentially complete $N$-colorable graph $\bar{G}$ extending $G$ such that $\bar{G}$ omits the type $\left\{\varphi\left(v_{1}, \mathbf{b}, d_{\beta}, \mathbf{e}\right)\right\} \cup\left\{E\left(v_{1}, d_{\alpha}\right): \alpha \in A^{\prime}\right\} \cup\left\{\neg E\left(v_{1}, d_{\alpha}\right): \alpha \in A\right.$ $\left.-A^{\prime}\right\}$. Since $c$ realizes this type in any $N$-colorable extension of $G^{\prime}, G^{\prime}$ cannot be embedded over $G$ in $\bar{G}$. Therefore $G^{\prime}$ is not a prime model extension of $G$ for $\left(T_{C C N}\right)_{U H}^{*}$.

Case II. Suppose the inverse image with respect to $f$ of each vertex of $K_{N}$ is infinite. Consider the collection $C=\{\theta(\mathbf{v}, \mathbf{b}): \theta$ is a conjunction of basic formulas defined in $G, G$ does not satisfy $\exists v \theta(v, b)$, but $\exists v \theta(v, b)$ is satisfied in some $N$-colorable extension of $G$ \}. $C$ is nonempty because $G$ is not existentially complete. Let $\theta_{0}(\mathbf{v}, \mathbf{b})$ be a formula in $C$ for which the number of distinct variables occurring in $\theta_{0}$ is minimal for all formulas in $C$. One may assume without loss of generality that $v_{1}$ occurs in $\theta_{0}$.

Suppose that $G^{\prime}$ is an existentially complete $N$-colorable graph extending $G$. Then $G^{\prime}$ satisfies $\exists v \theta_{0}(\mathbf{v}, \mathbf{b})$, since $G$ is an amalgamation base. Let $a_{1}, \ldots, a_{n}$ be vertices of $G^{\prime}$ for which $G^{\prime}$ satisfies $\theta_{0}(\mathbf{a}, \mathbf{b})$. The vertex $a_{1}$ is not in $G$ because of the minimality requirement in the choice of $\theta_{0}$. By supposition, there is an infinite set of vertices of $G$ such that each $N$-coloring of $G^{\prime}$ maps all these vertices to the same vertex of $K_{N}$ and maps $a_{1}$ to a different vertex of $K_{N}$, because all $N$-colorings of $G$ are equivalent to $f$ and all $N$-colorings of $G^{\prime}$ are equivalent (since $G^{\prime}$ is existentially complete). One may assume that the set $\left\{d_{\alpha}: \alpha \in A\right\}$ is such a set of vertices (otherwise replace this set by another suitable set). There is an element $\beta$ in $A$ for which $d_{\beta}$ is unequal to each of the vertices $a_{1}, \ldots, a_{n}, b_{1}, \ldots, b_{m}$, since $\left\{d_{\alpha}: \alpha \in A\right\}$ is 
an infinite set. Let $\theta_{1}\left(v_{1}, \mathbf{b}, d_{\beta}\right)$ be $\theta_{0}(\mathbf{v}, \mathbf{b}) \wedge E\left(v_{1}, d_{\beta}\right)$. The graph $G^{\prime \prime}$ obtained by adding an edge $E\left(a_{1}, d_{\beta}\right)$ to $G^{\prime}$ if $a_{1}$ is not adjacent to $d_{\beta}$ in $G^{\prime}$ is $N$-colorable, since $a_{1}$ and $d_{\beta}$ have distinct images under all $N$-colorings of $G^{\prime}$. Moreover, $G^{\prime \prime}$ satisfies $\theta_{1}\left(\mathbf{a}, \mathbf{b}, d_{\beta}\right)$, and $G^{\prime \prime}$ extends $G$. Thus, $\theta_{1}$ is in $C$.

The graph $G^{\prime}$ satisfies $\exists \mathbf{v} \theta_{1}\left(\mathbf{v}, \mathbf{b}, d_{\beta}\right)$, since $G$ is an amalgamation base and $G^{\prime}$ is existentially complete. Let $a_{1}^{\prime}, \ldots, a_{n}^{\prime}$ be vertices of $G^{\prime}$ for which $G^{\prime}$ satisfies $\theta_{1}\left(\mathbf{a}^{\prime}, \mathbf{b}, d_{\beta}\right)$. Let $A^{\prime}=\left\{\alpha: \alpha \in A\right.$ and $G^{\prime}$ satisfies $\left.E\left(a_{1}^{\prime}, d_{\alpha}\right)\right\}$. Then $\beta \in A^{\prime}$, so $A^{\prime}$ is nonempty. Let $\varphi\left(v_{1}, \mathbf{b}, d_{\beta}\right)$ be $\exists v_{2} \cdots \exists v_{n} \theta_{1}\left(\mathbf{v}, \mathbf{b}, d_{\beta}\right)$. The 1-type $\Delta=\left\{\varphi\left(v_{1}, \mathbf{b}, d_{\beta}\right)\right\} \cup\left\{E\left(v_{1}, d_{\alpha}\right): \alpha \in A^{\prime}\right\} \cup\left\{\neg E\left(v_{1}, d_{\alpha}\right): \alpha \in A-\right.$ $\left.A^{\prime}\right\}$ is not realized by any element of $G$ in any $N$-colorable extension of $G$, for no element of $G$ can satisfy $\varphi\left(v_{1}, \mathbf{b}, d_{\beta}\right)$ in any $N$-colorable extension of $G$ because of the minimality requirement in the choice of $\theta_{0}$. According to Lemma 2.18 , there is an existentially complete $N$-colorable graph $\bar{G}$ extending $G$ which omits the type $\Delta$. Since $a_{1}^{\prime}$ realizes $\Delta$ in all $N$-colorable extensions of $G^{\prime}, G^{\prime}$ cannot be embedded over $G$ in $\bar{G}$. Thus $G^{\prime}$ is not a prime model extension of $G$ for $\left(T_{C C N}\right)_{U H}^{*}$.

In either case $G$ would not have a prime model extension for $\left(T_{C C N}\right)_{U H}^{*}$ contrary to the initial supposition. Thus $G$ cannot be infinite.

3. Closing remarks. The theory of $N$-colorable graphs may be formalized in languages other than the one used in $\S \S 1,2$. An alternative, suggested by the practice of some graph theorists [1], would be a first order language with equality whose nonlogical symbols were two unary predicate symbols $V$ (for vertex) and $E$ (for edge) and a binary predicate symbol $P$ (for endpoint of an edge). The class of graphs would be the models of the theory $T_{G}$ with axioms

$$
\begin{aligned}
& \forall x[V(x) \leftrightarrow \neg E(x)] \\
& \forall v \forall x[P(v, x) \rightarrow(E(v) \wedge V(x))] \\
& \forall v[E(v) \rightarrow \exists x \exists y(P(v, x) \wedge P(v, y) \\
& \qquad \forall z[P(v, z) \rightarrow((z=x) \vee(z=y))])] .
\end{aligned}
$$

The class of graphs without loops or multiple edges is then axiomatized by' the theory $T_{G R E}$ consisting of the axioms of $T_{G}$ and axioms

$$
\begin{aligned}
\forall v[E(v) \rightarrow \exists x \exists y(\neg(x=y) & \wedge P(v, x) \wedge P(v, y))] \\
\forall v \forall x \forall y \forall z[(E(v) \wedge E(x) & \wedge P(v, y) \wedge P(v, z) \\
& \wedge P(x, y) \wedge P(x, z) \wedge \neg(y=z)) \rightarrow v=x] .
\end{aligned}
$$

Clearly, there is a natural one-to-one correspondence between the models of 
$T_{G R}$ and the models of $T_{G R E}$. A graph when considered as a model of $T_{G R E}$ will generally have more extensions, more subgraphs, and fewer homomorphic images than when it is considered as a model of $T_{G R}$.

The class of $N$-colorable graphs can be axiomatized by a theory $T_{G R E N}$ extending $T_{G R E}$. The theory $T_{G R E N}$ is decidable, but neither is it Horn nor is it finitely axiomatizable for $N>1$. The theory $T_{G R E N}$ does have a modelcompanion $T_{G R E N}^{*}$, which is not a model-completion. However, $T_{G R E N}^{*}$ is totally unrelated and dissimilar to $\left(T_{C C N}\right)_{U H}^{*}$. No graph is both a model of $\left(T_{C C N}\right)_{U H}^{*}$ when it is considered as a structure for $\left(T_{C C N}\right)_{U H}$ and a model of $T_{G R E N}^{*}$ when it is considered as a structure for $T_{G R E N}$. A graph is a model of $T_{G R E N}^{*}$ if and only if (1) it is infinite, and (2) the vertices can be partitioned into $N$ subsets each of which is infinite such that (i) no two vertices in the same subset are adjacent and (ii) each vertex $a$ is adjacent to each vertex $b$ which is not in the same subset as $a$. The reader may verify then that $T_{G R E N}^{*}$ is complete, decidable, has an effective elimination of quantifiers, is $\aleph_{0}$-categorical, and $\omega$-stable. For each infinite cardinal $\kappa$, the $N$-colorable graph of cardinality $\kappa$ which satisfies the conditions (1) and (2) above and each of whose subsets in the partition in (2) has cardinality $\kappa$ is saturated.

Appendix A. The following definitions of concepts from universal algebra and [9] are included here for the convenience of the reader.

Let $T$ be a universal theory.

Definition 1. A model $\mathfrak{M}$ of $T$ generated by elements $a_{1}, \ldots, a_{n}$ is finitely presented if there is a conjunction $\varphi(v)$ of atomic formulas such that (i) $\mathfrak{M}$ satisfies $\varphi(\mathbf{a})$ and (ii) if $\mathfrak{N}$ is a model of $T$ generated by elements $b_{1}, \ldots, b_{n}$ and $\mathfrak{R}$ satisfies $\varphi(\mathbf{b})$ then there is a homomorphism from $\mathfrak{M}$ onto $\mathfrak{R}$ which sends $a_{i}$ to $b_{i}$ for $i=1, \ldots, n$. The formula $\varphi$ is then called a finite presentation for $\mathfrak{M}$.

DEFINITION 2. The theory $T$ has finite presentations if each conjunction $\varphi(v)$ of atomic formulas for which $\exists \mathrm{v} \varphi$ is consistent with $T$ is a finite presentation of some model of $T$.

Every universal Horn theory has finite presentations.

Definition 3. A conjunction $\varphi(v)$ of atomic formulas has a finite presentation decomposition if there is a collection $\mathfrak{M}_{1}, \ldots, \mathfrak{M}_{k}$ of finitely presented models of $T$ where $\mathfrak{M}_{j}$ is generated by $a_{1}^{j}, \ldots, a_{n}^{j}$ such that (i) $\mathfrak{M}_{j}$ satisfies $\varphi\left(\mathbf{a}^{j}\right)$ for $i=1, \ldots, k$, and (ii) if $\mathfrak{N}$ is a model of $T$ generated by elements $b_{1}, \ldots, b_{n}$ and $\mathfrak{R}$ satisfies $\varphi(\mathbf{b})$, then there is a $j$ between 1 and $k$ inclusive and a homomorphism from $\mathfrak{M}_{j}$ onto $\mathfrak{N}$ which sends $a_{i}^{j}$ to $b_{i}$ for $i=1, \ldots, n$.

Definition 4. The theory $T$ has finite presentation decompositions if each conjunction $\varphi(v)$ of atomic formulas for which $\exists v \varphi$ is consistent with $T$ has a finite presentation decomposition. 
Definition 5. The theory $T$ is locally finite if each finitely generated model of $T$ is finite.

DEFINITION 6 . The theory $T$ is coherent if each finitely generated submodel of a finitely submodel of a finitely presented model of $T$ is finitely presented itself.

Definition 7. Assume that $\psi(\mathbf{v})$ is a universal formula, $\mathfrak{M}$ is a model of $T$, and $a_{1}, \ldots, a_{n}$ are elements of $\mathfrak{M}$. The sentence $\psi(a)$ is persistable over $\mathfrak{M}$ if there is an open formula $\varphi(\mathbf{v}, \mathbf{x})$ such that $\forall \mathbf{x}(\varphi(v, x) \rightarrow \psi(\mathbf{v}))$ is deducible from $T$ and $\exists x \varphi(a, x)$ is satisfied in some extension of $\mathfrak{M}$ which is a model of $T$.

Definition 8. The theory $T$ has the universal conservative homomorphism lifting property for finite presentations if, whenever a finitely presented model $\mathfrak{M}$ of $T$ with generators $a_{1}, \ldots, a_{n}, b_{1}, \ldots, b_{m}$ satisfies $\chi(\mathbf{a}, \mathbf{b})$ for a conjunction $\chi(\mathbf{v}, \mathbf{x})$ of negated atomic formulas and $\mathfrak{N}$ is the submodel of $\mathfrak{M}$ generated by $a_{1}, \ldots, a_{n}$, there is a universal formula $\psi(v)$ such that

(i) $T \cup \operatorname{PDiag}(\mathfrak{M}) \vdash \chi(\mathbf{a}, \mathbf{b}) \rightarrow \psi(\mathbf{a})$, and

(ii) if $h$ is a homomorphism from $\mathfrak{N}$ into a $a$ model $\mathfrak{N}^{\prime}$ of $T$ and $\psi\left(h\left(a_{1}\right), \ldots, h\left(a_{n}\right)\right)$ is persistable over $\mathfrak{R}^{\prime}$, then there is a model $\mathfrak{M}^{\prime}$ of $T$ which extends $\mathfrak{N}^{\prime}$ and a homomorphism $\bar{h}$ from $\mathfrak{M}$ into $\mathfrak{M}^{\prime}$ such that $\bar{h}\left(a_{i}\right)=h\left(a_{i}\right)$ for $i=1, \ldots, n$ and $\mathfrak{M}^{\prime}$ satisfies $\chi\left(h\left(a_{1}\right), \ldots, h\left(a_{n}\right)\right.$, $\left.\bar{h}\left(b_{1}\right), \ldots, \bar{h}\left(b_{m}\right)\right)$.

THEOREM A. If a universal theory with finite presentation decompositions is coherent and has the universal conservative homomorphism lifting property for finite presentations, then it has a model-companion.

Theorem $\mathbf{A}$ is a special case of one part of Theorem 1 of [9].

NOTE ADDED IN PROOF. Theorems 2.2 and 2.12 are special cases of more general results proved by S. Burris and $\mathrm{H}$. Werner (Sheaf Constructions and Their Elementary Properties, to appear in this journal). S. Burris has subsequently generalized Theorem 2.8 as well (Model Companions for Finitely Generated Universal Horn Classes).

\section{BIBLIOGRAPHY}

1. C. Berge, Graphs and hypergraphs, North Holland, Amsterdam, 1973.

2. S. Feferman and R. L. Vaught, The first order properties of products of algebraic systems, Fund. Math. 47 (1959), 57-103.

3. J. Hirschfeld and W. H. Wheeler, Forcing, arithmetic, division rings, Lecture Notes in Math., vol. 454, Springer-Verlag, Berlin and New York, 1975.

4. H. J. Keisler, Six classes of theories, J. Austral. Math. Soc. 21 (1976), 257-266.

5. G. Ringel, Färbungsprobleme auf Flächen und Graphen, VEB Deutscher Verlag der Wissenschaften, Berlin, 1959. 
6. A. Robinson, Introduction to model theory and to the metamathematics of algebra, NorthHolland, Amsterdam, 1965.

7. S. Shelah, Stability, the f.c.p., and superstability; model theoretic properties of formulas in first order theory, Ann. Math. Logic 3 (1971), 271-362.

8. J. R. Shoenfield, Mathematical logic, Addison-Wesley, Reading, Mass., 1967.

9. W. H. Wheeler, A characterization of companionable, universal theories, J. Symbolic Logic 43 (1978), 402-429.

10. W. Taylor, Atomic compactness and graph theory, Fund. Math. 65 (1969), 139-145.

Departiment of Mathematics, Indana University, Bloomington, IndinNa 47401 